\title{
Scan to BIM: the development of a clear workflow for the incorporation of point clouds within a BIM environment
}

\author{
R. Laing ${ }^{1}$, M. Leon ${ }^{1}$, J. Isaacs ${ }^{2} \&$ D. Georgiev ${ }^{1}$ \\ ${ }^{I}$ Scott Sutherland School of Architecture and Built Environment, \\ Robert Gordon University, UK \\ ${ }^{2}$ School of Computing Science and Digital Media, \\ Robert Gordon University, UK
}

\begin{abstract}
The emergence, in recent years, of technology to support the use of data rich models within architecture, has significantly aided the uptake of building information modelling. Simultaneously, there has been a rapid expansion in the capabilities and widespread use of 3D high definition laser scanning technology. Although laser scanning has often been associated with industries outside of architecture and building (including heavy engineering and oil and gas installations), the potential to record the existing built environment is clear. Indeed, well-established concepts within building and materials conservation concerning the importance of being able to accurately monitor and recognise the importance of surface characteristics, are well suited to the use of scanning to capture geometrical idiosyncrasies as well as designed detail. Likewise, the ability to capture structures which are well outside the physical reach of the expert, makes the accurate recording of large scale buildings and streetscapes possible, and at a speed and level of accuracy which was not feasible even 15 years ago. This paper concerns a series of workflow stages which are required to incorporate the output of laser scan data within a BIM environment. Although it is possible to import point clouds within industry standard BIM software, in order to make best use of the highly accurate and often massive data files a certain amount of postprocessing and modelling is required. We describe a process whereby cloud data can be transformed to produce representative surface meshes, and explore how the resultant models can be linked with meta data within the BIM environment. The development of methods to help the incorporation of already existing
\end{abstract}


environments within BIM will be of great value within FM, building conservation and new design alike. Therefore, the refinement and adoption of clear methods to support such work is a vital step towards BIM maturation.

Keywords: BIM scanning, models contours, 3D scanning, BIM.

\section{Introduction: previous work}

\subsection{Technology and 3D models reconstruction for BIM}

3D models acquisition produced from laser scanners and photogrammetry provide content rich information of the as-built conditions. They are becoming a standard practice in construction, heritage conservation and facilities management, within a range of different industries, from oil and gas and heavy engineering to AEC industry. Relevant developed technologies include photogrammetry, image based sensing technologies and 3D remote sensing technologies, like Global Positioning systems (GPS), Global Navigation Satellite System, Radio Frequency Identification (RFID) and Laser Detection and Ranging (LIDAR) [1-3]. Even though the produced data from the aforementioned methods include potential for their incorporation to more advanced data viewing or processing systems and software, limited progress has been observed regarding the automated process of that data. As a result, significant research has been monitored on developing processes and algorithms for processing 3D data.

Previous research on reconstruction of as-built BIM models from 3D scanned data showcase the difficulties with transferring the information in a fast and accurate way. Tang et al. [4] describe how the manual methods of transferring the information to BIM are time-consuming, prone to errors and eventually lack accuracy.

Manual creation of BIMs initiated with 3D data collection through laser scanners followed by pre-processing, or else data alignment and registration, with manual removal of unwanted data and possible conversion in surface data/triangular mesh. The actual creation of BIMs according to the $3 \mathrm{D}$ scanned data is comprised of establishing topological relationships among the building components, modeling the geometric components based either on cross sections with surface extrusion, or on fitting geometric primitives to the 3D data. However, a range of problems have been observed with this method, including issues with transferring the models across a range of software, thus data interoperability problems, and lack of accuracy when transferring the data.

As a result, relevant and developing research for automated $3 \mathrm{D}$ data reconstruction suggests a range of methods including subdividing the overall process into geometric modelling, object recognition and object relationship modeling for transferring 3D measurements into as-built BIMs [4]. These methods can be further subdivided into a number of automated processes like shape representations [5], relationship representations [6], automated geometric modelling [7], planar and curved surfaces modelling, object recognition and relationships modelling. 


\subsection{Heritage 3D scanning}

This paper focuses on the automated geometric modelling, especially in relation to scanning heritage sites. Laser scanning has been developing into a standard tool for cultural heritage and research applications have been ranging from heritage preservation and site conservation to architectural heritage [8-10]. Sites and structures, statues and monuments with historic significance can be monitored in their existing situation, hence allowing relevant professionals and organisations to keep a record of their evolution over time. What is more, the case studies described in this paper are related to the city of Aberdeen and the town of Elgin since they are urban integrated monuments and sites that require further understanding in relation to the impact of changes in the city to the relevant heritage.

\section{Case studies}

The surface based non-parametric geometric modelling or surface reconstruction is the applied method during the 3D scanned data analysis and meta-processing. Relevant research in the field is related to geometric surface reconstructions [11, 12], where the 3D point clouds are translated into polyhedral solids involving "the shape changes of an object at the boundary of the mesh growing area and to create a priority queue to the advancing front of the mesh area according to the changes. The mesh growing process is then driven by the priority queue for efficient surface reconstruction" [11].

The case studies presented here are interesting in that they collectively demonstrate a progression of workflows, including the use of scan data as a 'template', the use of raw data to help produce virtual 'surface models' suitable for use as objects within BIM software, and the extraction of surface data (contour) in relation to an urban park.

The workflow concludes with the extraction of models using a laser cutter, in an attempt to bridge between virtual and tactile modelling.

\subsection{Environmental modelling}

The first case study described in this paper was derived from a scanning project undertaken to record a complex urban area, which included both natural landscape and examples of the built environment dating back many centuries. Although the initial purpose of the scanning activity was to record a monument located at the top of an urban hill, the capabilities of the scanning equipment are such that the range and detail of the resulting point cloud also incorporated data pertaining to a number of municipal buildings constructed from the early 1800 s up to the late 1970s. Streetscapes 3D scanning had a high level of complexity, it required a great number of scanning positions that incorporated rich architectural details, different natural elements and multiple street levels. The process of the specific project is described in detail in [13] and it is illustrated in Figure 1.

The specific research made use of a Leica C10 3D scanner (Figure 2), for providing a rapid and accurate understanding of the built environment, not only in 


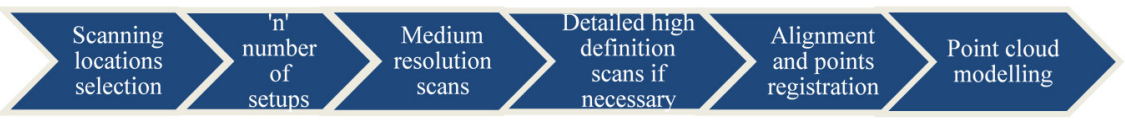

Figure 1: 3D scanning of urban environments.

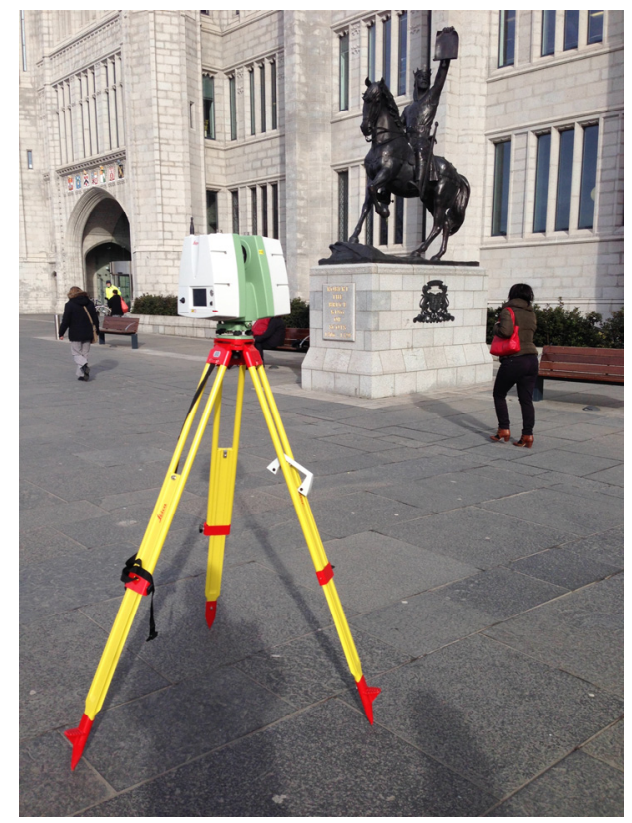

Figure 2: Scanning statue (Leica C10 scanner).

relation to buildings but also with a focus on monuments and landmark. The particular scanner is suitable both for interior and exterior scanning with high definition scanning capabilities, and has a scanning range of up to $300 \mathrm{~m}$ [14]. The end result consists a point cloud that represents the collected (scanned) points without connecting them or creating a 3D model or mesh. The collected data are dense, precise and highly accurate, hence allowing for comprehensive acquisition of range point clouds.

Whilst the scan information relating to the older buildings and monuments located within the site was extremely complex (due to irregular surfaces and a vernacular and unplanned approach to the arrangement of buildings), an obvious way in which the process of converting scanned data into a format usable within a BIM software environment was suggested by the geometrically regular, and rectangular, design of the municipal structures. The first example is a 3D scan of a main street in the town of Elgin in Scotland.

The particular data comprised of the 3D scan output, the point clouds. Although high in accuracy, the data lacked information pertaining to the geographical location of the data, or the height of the structures in relation to standard horizontal 
points. Therefore, it was essential to insert horizontal levels to which the BIM model itself could refer to. Figure 3 illustrates the integration of buildings and structures as captured with the laser scanner followed by data rich representative elements, which in this case are Revit developed models of the scanned environment. It is important to acknowledge that during this process a significant degree of knowledge on the part of the building model and also on the part of model's user was required.

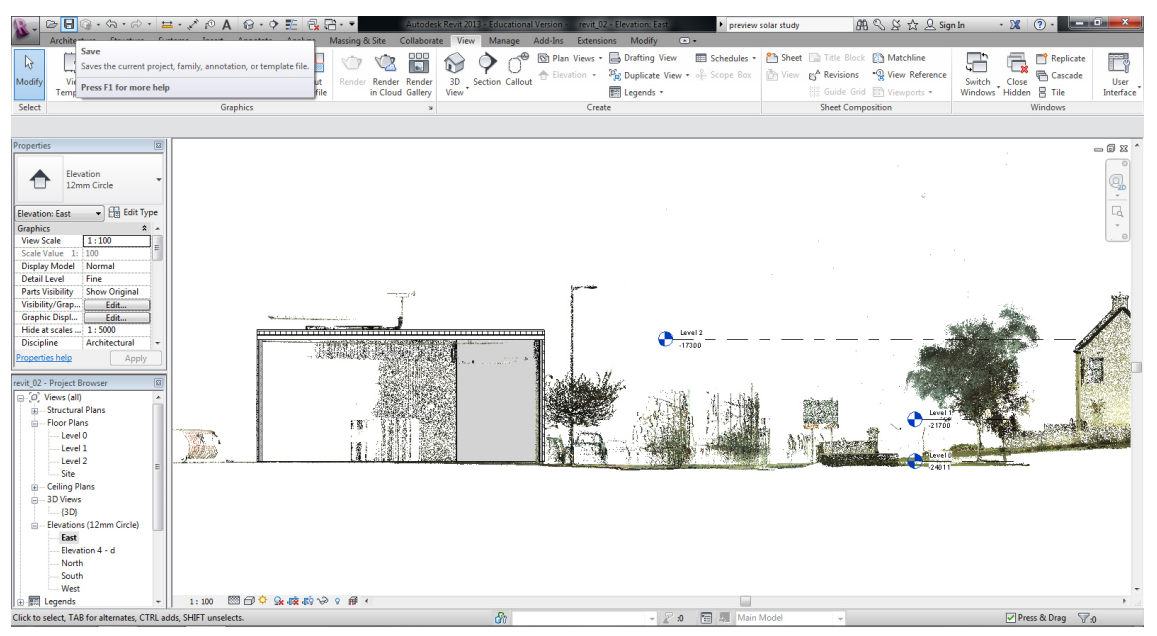

Figure 3: Scan data imported to Autodesk REVIT, for use as a template.

\subsection{Surface and solid modelling}

A second project, following the environmental modelling, focused on scanning architectural heritage in the city of Aberdeen. During this project, the researchers were engaged in developing geometrically accurate virtual models of six statues located in the city centre. The intended use was to provide information for a public exhibition for displaying the statues and highlighting them within the urban context. Therefore, the focus was on specific monuments and not on navigable space as the previous environmental modelling project was.

A thorough scanning process was followed, with at least four separate setups required for each statue, in order to ensure that all the information was collected. A brief description of the process is as follows: after the scanning locations selection and the separate setups, for each position a low resolution scan took place initially, followed by a high resolution one. This information was imported to the scanner software, where the identification of common points between scans took place, where it became possible to establish the relative position of each scan, a process called alignment and points registration. The point cloud data was analysed afterwards for surface modelling and developed into solid meshes, while the process is depicted in Figure 4. 


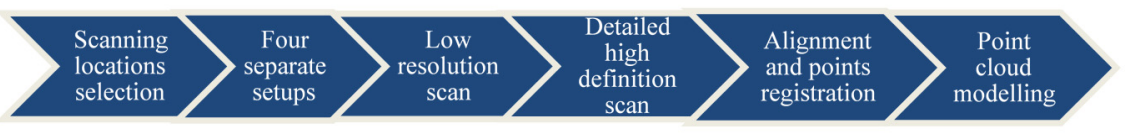

Figure 4: 3 D scanning of monuments.

Although solid meshes can have less detail than the original point cloud, they have the benefit of not deteriorating when viewed in an interactive context. From a first person's perspective, when a user or environment camera approaches an object in a point cloud it will gradually dissolve into constituent points. This breakdown can reduce the realism of the environment or model for a non-expert viewer. In contrast a solid mesh will retain its solid appearance, although the texture resolution may be reduced [12]. Figure 5 shows how the appearance of the horse's head changes between the two techniques.

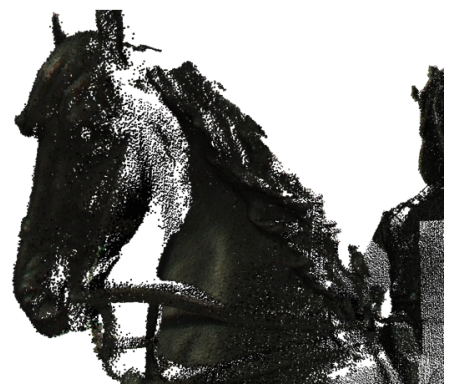

(a)

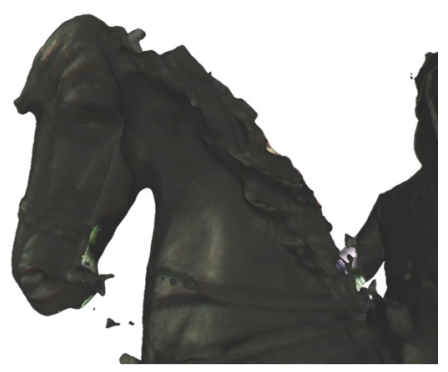

(b)

Figure 5: Point cloud and solid mesh representation. (a) High detailed point cloud, dissolves on approach. (b) Lower detailed solid mesh, retains solidity but loses definition.

The first stage in generating a solid mesh from a 3D scan is sub-sectioning and cleaning. Generally the point cloud will contain a fair degree of noise. Excess noise will prevent an accurate solid mesh being created as algorithms used are, for the most part, unable to discriminate between the clusters of noise and solid surfaces. This noise can come from a variety of sources, particularly in urban environments where people, vehicles and even precipitation will cause partial shadows or point clusters within the cloud data. Figure 6 shows the impact of snow in the centre of the scan on the noise present within the scene.

Once the cloud has been cleaned and isolated it can be imported into the Meshlab software (http://meshlab.sourceforge.net/) where the generation of the solid mesh is performed. Meshlab contains a number of methods that allow us to further clean, simplify, extract and generate surfaces for our point cloud.

The point cloud can be simplified using Poisson Disk Simplification. This allows a target number of points to be set to which Meshlab will try to reduce the point cloud. A Poisson disk point set is a uniformly distributed set of points in which no two points are closer to each other than a given minimum distance [6]. 


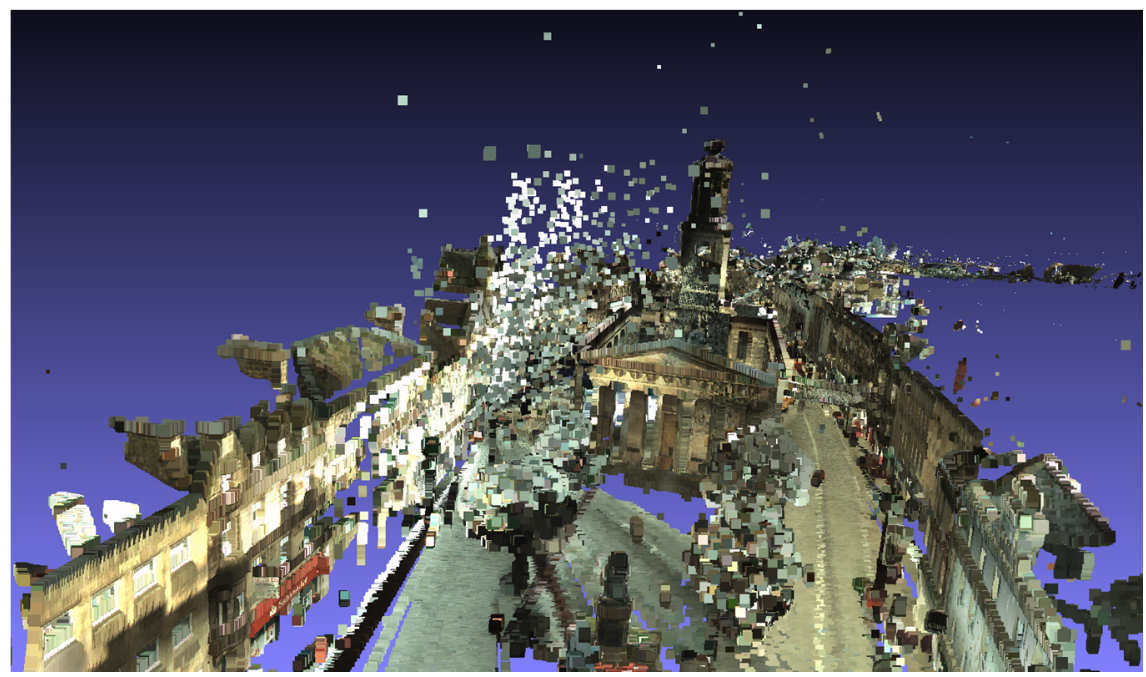

Figure 6: Noise, in this case snow, must be removed before surface creation.

This allows the overall shape of the cloud to be retained whilst reducing the number of points. To generate a surface we need to calculate the normals for the points in our cloud to ensure that the resulting surface is orientated in the correct direction. Meshlab provides a method to compute the normals based on the neighbouring points. The setting used for the neighbourhood will depend entirely on the quality of the scan and the type of object being represented. If for example it was a large flat surface, a large neighbourhood would be most efficient. For a higher detailed object, like the statues, a smaller neighbourhood of around 15-20 will create a truer representation of statue, whilst smoothing out the majority of the noise.

Poisson Surface Reconstruction can then be used to create a surface between points that are a specific distance apart, creating a solid surface over the point cloud. The Poisson algorithm takes a number of parameters which define how the surface creation is performed. The Octree-Depth affects the detail of final mesh, a higher number here will provide a more detailed but more complex result and will take much longer, and higher processing power, to create. The Solver-Divide parameter allows for some saving in memory whilst mesh processing. Finally, the Sample per Node parameter determines how many points are calculated as a node, generally smoothing the surface and again removing some noise and detail. At this stage, fine detail can be lost since the Poisson algorithm cannot distinguish between residual noise, fine surfaces and fine detail. The image below shoes how the parchment the statue is holding is lost in the final mesh as the point cloud representing this object had tightly packed points which were ultimately smoothed out. The difference can clearly be seen when comparing the original statue with the solid mesh in Figure 7, below. This can be solved by creating a number of surfaces that use different Poisson parameters and merging the results until the desired detail is achieved. 


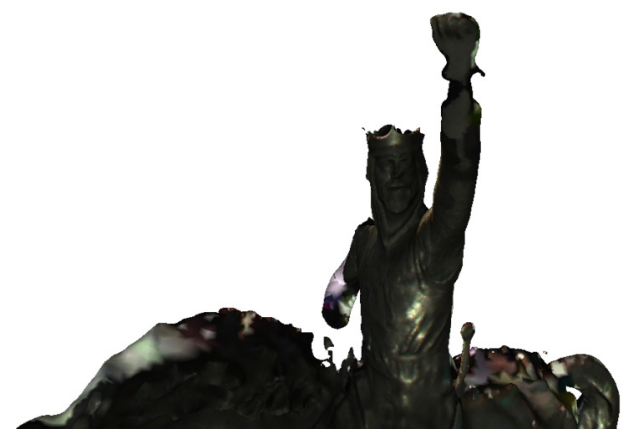

Figure 7: Untextured mesh, highlighting missing structures due to smoothing.

The resultant solid mesh at this stage (Figure 7) is untextured. The Leica scanner is able to capture full colour from the original scene and store this in the point cloud. Using parameterisation we can transfer the colour from the original cloud to our new solid mesh. Because the mesh is a simplified version of the point cloud it will not contain an exact location for every point in the cloud, parameterisation will apply texture coordinates from the original cloud to the nearest possible point on our new mesh giving us a detailed texture that reflects the original scanned scene as illustrated in Figure 8.

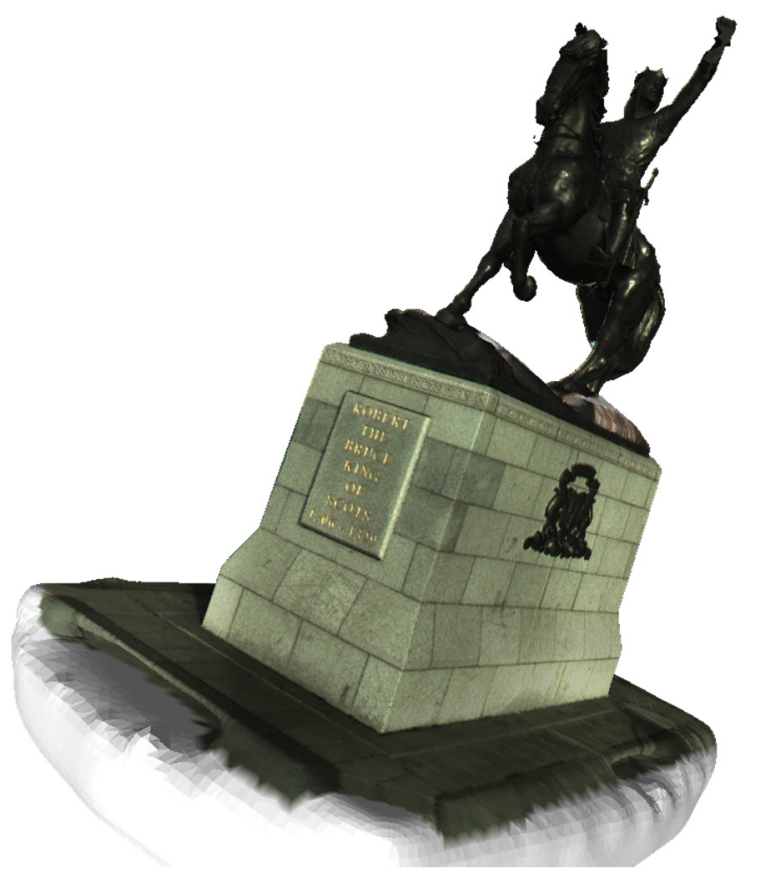

Figure 8: Statue converted to surface model. 


\subsection{Extraction of contour data}

A final approach to analyse and develop 3D scanned data into a BIM model includes the extraction of contour data, which can be utilised afterwards for 3D milling and cutting. The specific approach is a meta-analysis of the environmental modelling process from the site in Elgin. During the specific project, an assembled point cloud was imported to Revit, which was considered as a unified object from the software, as depicted in Figure 9.

Afterwards, a number of sections were drawn in the model, with 1 meter distance in between them, thus slicing the contour lines of the site (Figure 10). The plans acquired from this process provided the descriptions of the site's topography and it became possible to 'draw around the dots' and obtain the different levels as layers. A post production process followed, where these lines where inserted into different types of software in a proper scale and the layers/contour lines became recognisable. Following the particular output, the drawings were exported back to AutoCAD and prepared for laser cutting. Eventually, the end result is presented in Figure 11.

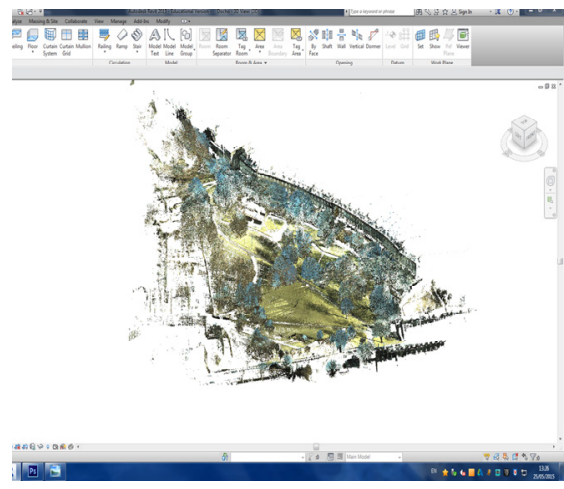

Figure 9: Environmental modelling imported into Revit.

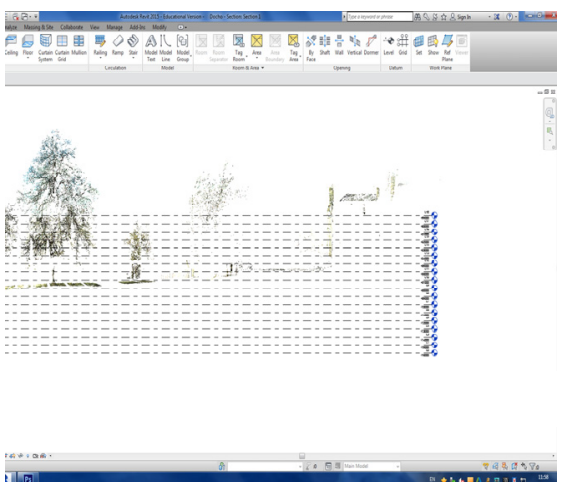

Figure 10: Addition of level indicators within RIVIT.

\section{Conclusions and further research}

The case studies presented here highlight that although it is not possible to fully automate the process of incorporating the output of laser scan data within a BIM environment, it is still possible to break down this process into a number of automated steps. Some of the steps in each of the work flows do still require some knowledge of the structure being scanned and there is also a degree of subjectiveness in the quality of the output. However, it is argued that if the workflows demonstrated here are followed, they will speed up and improve the accuracy of 3D scanned data transfer for meta-processing. The presented techniques work on a range of different environments from smaller city features such as the statues to larger city scapes and land forms. 


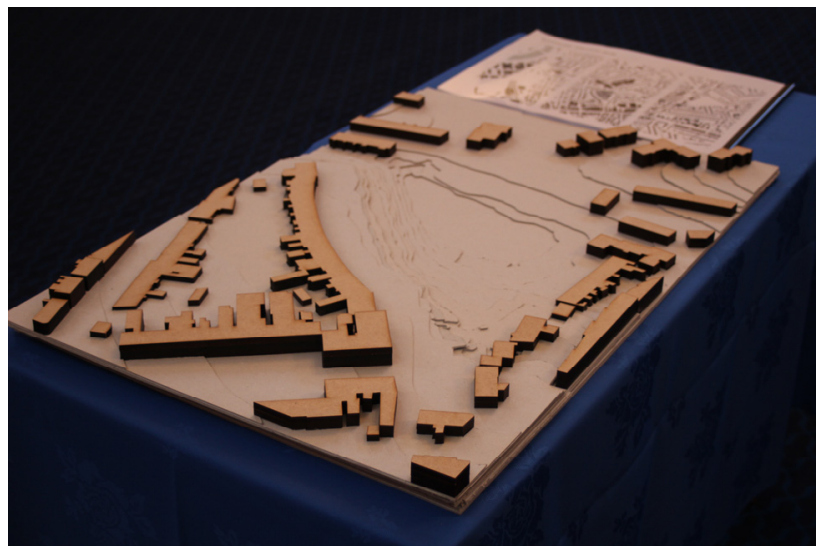

Figure 11: Model of the environmental scanning data.

Laser scan data provide very highly accurate representations of real world environments without the need for complex 3D modelling and object generation. The complexity of the point clouds currently precludes their automated incorporation into BIM environments whilst still maintaining all the detailed information they contain. As user engagement in planning and design increases it is likely that laser scans will be used in more non-standard environments, such as game engines. Further research is therefore required to develop more automated work flows that require less user interaction, whilst still maintaining control over the resulting output.

\section{References}

[1] M. Golparvar-Fard, J. Bohn, J. Teizer, S. Savarese and F. Peña-Mora, "Evaluation of image-based modeling and laser scanning accuracy for emerging automated performance monitoring techniques," Autom. Constr., vol. 20, pp. 1143-1155, 12. 2011.

[2] F. Bosché and C.T. Haas, "Automated retrieval of 3D CAD model objects in construction range images," Autom. Constr., vol. 17, pp. 499-512, 5. 2008.

[3] F. Bosché, M. Ahmed, Y. Turkan, C.T. Haas and R. Haas, "The value of integrating Scan-to-BIM and Scan-vs-BIM techniques for construction monitoring using laser scanning and BIM: The case of cylindrical MEP components," Autom. Constr., vol. 49, Part B, pp. 201-213, 1. 2015.

[4] P. Tang, D. Huber, B. Akinci, R. Lipman and A. Lytle, "Automatic reconstruction of as-built building information models from laser-scanned point clouds: A review of related techniques," Autom. Constr., vol. 19, pp. 829-843, 11. 2010.

[5] N. Amenta, S. Choi and R.K. Kolluri, "The power crust, unions of balls, and the medial axis transform," Computational Geometry, vol. 19, pp. 127-153, 7. 2001. 
[6] H. Hoppe, T. DeRose, T. Duchamp, J. McDonald and W. Stuetzle, "Surface reconstruction from unorganized points," in 19th Annual ACM Conference on Computer Graphics and Interactive Techniques - SIGGRAPH 1992; Chicago, IL, USA, pp. 71-78, 1992.

[7] Cline, D., Jeschke, S., White, K., Razdan, A., \& Wonka, P. (2009, June). Dart throwing on surfaces, Computer Graphics Forum, vol. 28, no. 4, pp. 1217-1226. Blackwell Publishing Ltd.

[8] A. Hakonen, J. Kuusela and J. Okkonen, "Assessing the application of laser scanning and 3D inspection in the study of prehistoric cairn sites: The case study of Tahkokangas, Northern Finland," Journal of Archaeological Science: Reports, vol. 2, pp. 227-234, 6. 2015.

[9] S. Al-kheder, Y. Al-shawabkeh and N. Haala, "Developing a documentation system for desert palaces in Jordan using 3D laser scanning and digital photogrammetry," Journal of Archaeological Science, vol. 36, pp. 537-546, 2. 2009.

[10] K. Lambers, H. Eisenbeiss, M. Sauerbier, D. Kupferschmidt, T. Gaisecker, S. Sotoodeh and T. Hanusch, "Combining photogrammetry and laser scanning for the recording and modelling of the Late Intermediate Period site of Pinchango Alto, Palpa, Peru," Journal of Archaeological Science, vol. 34, pp. 1702-1712, 10. 2007.

[11] X. Li, C. Han and W.G. Wee, "On surface reconstruction: A priority driven approach,” Comput.-Aided Des., vol. 41, pp. 626-640, 9. 2009.

[12] Fabio, R. (2003). From point cloud to surface: the modeling and visualization problem. International Archives of Photogrammetry, Remote Sensing and Spatial Information Sciences, 34(5), W10.

[13] R. Laing, M. Leon, L. Mahdjoubi and J. Scott, "Integrating Rapid 3D Data Collection Techniques to Support BIM Design Decision Making," Procedia Environmental Sciences, vol. 22, pp. 120-130, 2014.

[14] Leica ScanStation C10. 04/04, http://www.leica-geosystems.co.uk/en/ Leica-ScanStation-C10_79411.htm accessed April 2015. 